\title{
High temperature thermoelectric properties of PbTe prepared by high pressure method
}

\author{
Haotian Fan ${ }^{1}$, Taichao $\mathrm{Su}^{1 *}$, Hongtao $\mathrm{Li}^{2}$, Youjin Zheng ${ }^{3}$, Shangsheng Li ${ }^{1}$, \\ Meihua Hu ${ }^{1}$, Hongan MA ${ }^{4}$, XiaOpeng Jia ${ }^{4}$ \\ ${ }^{1}$ Institute of Materials Science and Engineering, Cultivating Base for Key Laboratory of Environment-friendly Inorganic \\ Materials in University of Henan Province, Henan Polytechnic University, Jiaozuo 454000, China \\ ${ }^{2}$ Shanghai Entry-Exit Inspection \& Quarantine Bureau, Shanghai 200135, China \\ ${ }^{3}$ Department of Physics, Mudanjiang Normal College, Heilongjiang Province Key laboratory of New Carbon-base Functional \\ and Superhard Material, Mudanjiang 157011, China \\ ${ }^{4}$ National Lab of Superhard Materials, Jilin University, Changchun 130012, China
}

\begin{abstract}
Thermoelectric materials PbTe were synthesized by high pressure method. The microstructure and temperature dependence of thermoelectric properties were studied. Some second phases with nano-structure were found in the high pressure synthesized samples. Compared with that of PbTe prepared at an atmospheric pressure, the samples prepared by high pressure method had smaller grain sizes, lower resistivity and lower phonon thermal conductivity. The maximum figure of merit, $\mathrm{Z}$ reached $8.86 \times 10^{-4} \mathrm{~K}^{-1}$ for the sample prepared at $4 \mathrm{GPa}$. This value was about twice times higher than that of the sample prepared at ambient pressure and it matched to that of highly doped $\mathrm{PbTe}$.
\end{abstract}

Keywords: thermoelectric material; high pressure; PbTe

(C) Wroclaw University of Technology.

\section{Introduction}

Thermoelectric (TE) materials as a kind of semiconductor materials are gradually developed based on Seebeck effect, Peltier effect and Thomson effect. They can convert thermal energy into electrical energy directly and vice versa [1]. Hence, TE materials are applied to manufacturing power generation and cooling devices [2]. Compared with the traditional power generation and cooling devices, the TE devices have many advantages, such as light weight, small size, lack of any moving part and pollution-free operation [1]. Therefore, TE materials have a good application foreground in the field of heat recovery from industrial wastes and space exploration.

The properties of TE materials are determined by the figure of merit, $Z=S^{2} \sigma / \kappa$, where $S$ is the Seebeck coefficient, $\sigma$ is the electrical conductivity

*E-mail: taichao80@hotmail.com and $\mathrm{K}$ is the thermal conductivity [3]. As seen from the formula, the TE materials with high properties must have a large Seebeck coefficient, a low thermal conductivity and a high electrical conductivity. The large Seebeck coefficient ensures the obvious thermoelectric effect. The small thermal conductivity keeps heat near the joint and the high electrical conductivity ensures minimizing the joule heat [3]. The electrical properties of TE materials are usually expressed by the power factor, defined as $S^{2} \cdot \sigma$ or $S^{2} / \rho$, where $\rho$ is electrical resistivity. It can be optimized by tuning the carrier concentration. The total thermal conductivity $k$ consists of two parts, the electronic thermal conductivity $\kappa_{\mathrm{e}}$ and the phonon thermal conductivity $\kappa_{\text {ph }}$.

Compared with other methods of the preparation of thermoelectric materials, the high pressure method has many advantages, such as restraining disorders, phase separation and other complicating factors during the preparation of the materials [4]. In the literature there are some reports on $\mathrm{PbTe}$ 
prepared by high pressure method (high pressure and high temperature, high pressure sintering) [57]. But these reports focus on the thermoelectric properties below room temperature. As it is well known, $\mathrm{PbTe}$ is a kind of a good thermoelectric material in a temperature range of 400 to $800 \mathrm{~K}$ [8]. Up to now, there has been no report on high temperature thermoelectric properties of $\mathrm{PbTe}$ prepared by high pressure method.

In this paper, thermoelectric material $\mathrm{PbTe}$ was prepared by high pressure method. The temperature-dependent thermoelectric properties were studied from room temperature to $600 \mathrm{~K}$.

\section{Experimental}

The PbTe samples were prepared from $\mathrm{Pb}$ $(99.9 \%)$ and $\mathrm{Te}(99.999 \%)$ powders as the sources, which were weighed according to the stoichiometry. After being uniformly mixed in an agate mortar, the mixtures were shaped by press. The rod shaped samples were assembled for high pressure synthesis. The samples were prepared in a cubic anvil high pressure apparatus (SPD $6 \times 1200$ ) with a sample chamber of $23 \mathrm{~mm}$ length. The temperature $(1200 \mathrm{~K})$ and pressure $(2$ and $4 \mathrm{GPa})$ were kept for 30 minutes. The pressure was controlled by the oil press load, which was calibrated by the pressure induced phase transitions of bismuth, thallium and barium metals. The temperature was estimated by the relationship of input heater power and temperature, which was measured by the platinum-rhodium thermocouples. For comparison, the $\mathrm{PbTe}$ sample was also prepared at normal pressure $(0 \mathrm{GPa})$. The method of synthesis could be found in the references $[6,7]$. Before testing, all the samples were annealed at $600 \mathrm{~K}$ for $1 \mathrm{~h}$ under vacuum condition.

The samples were cut and polished on the surface for thermoelectric measurements, which were carried out at a high temperature. X-ray diffraction (XRD) measurements with $\mathrm{Cu}-\mathrm{K} \alpha$ radiation were carried out on an X-ray diffractometer (D/MAXRA). The fracture surface morphology was characterized by a HITACHI S-4800 scanning electron microscope (SEM). The Seebeck coefficient
$S$ and electrical resistivity $\rho$ at different temperatures, from room temperature to $600 \mathrm{~K}$, were measured simultaneously with a LRS-3 apparatus. The thermal conductivity $K$ was calculated by using $\kappa=D \rho C_{P}$, where $\rho$ is the sample density measured by the Archimedes method. The thermal diffusivity D was measured on a Netzsch LFA457 laser flash apparatus.

\section{Results and discussion}

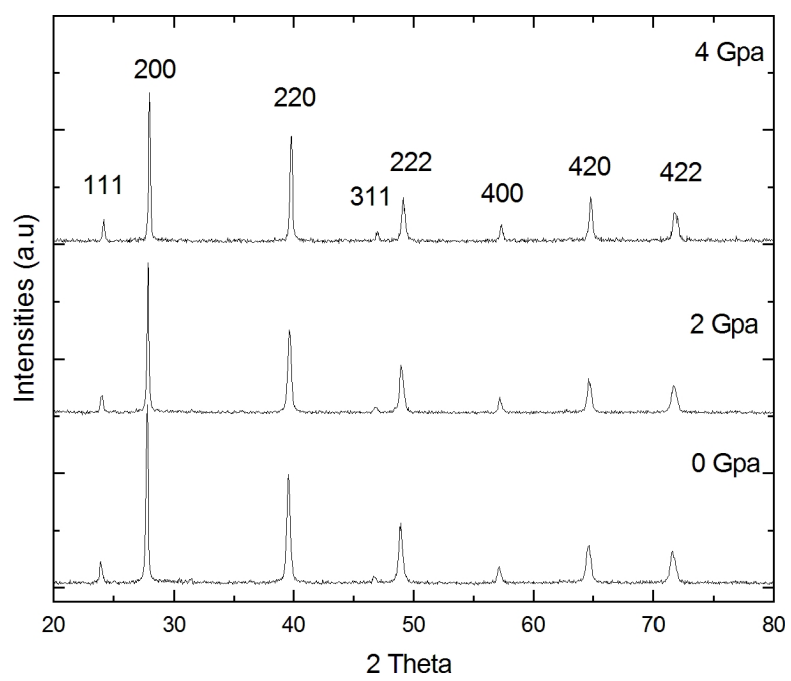

Fig. 1. XRD patterns of PbTe prepared under different pressures.

The XRD spectra of PbTe prepared at $0 \mathrm{GPa}$, $2 \mathrm{GPa}$ and $4 \mathrm{GPa}$ are shown in Fig. 1. The patterns indicate that all the samples are of NaCl-type structure and no noticeable second phases are detected. The lattice constant of PbTe prepared at $0 \mathrm{GPa}$ is about $6.455 \AA$, which is close to the value reported by Pei et al $(0.6454 \mathrm{~nm})$ [9]. As seen from Table 1 , the lattice constant of PbTe decreases slightly with an increase of pressure. It indicates that the atom spacing for PbTe changes slightly under high pressure. The density increases with an increase in pressure, which may be helpful to decrease the resistivity of PbTe.

Fig. 2 shows the SEM microphotographs of fractured surface for the PbTe prepared at ambient and high pressure (the SEM of sample prepared at $2 \mathrm{GPa}$ is nearly the same as that prepared at 
Table 1. Lattice constant, relative density and phonon thermal conductivity of $\mathrm{PbTe}$.

\begin{tabular}{cccc}
\hline $\begin{array}{c}\text { Synthesis } \\
\text { pressure }(\mathrm{GPa})\end{array}$ & $\begin{array}{c}\text { Lattice } \\
\text { constant }(\AA)\end{array}$ & $\begin{array}{c}\text { Density } \\
\left(\mathrm{g} / \mathrm{cm}^{3}\right)\end{array}$ & $\begin{array}{c}\text { Phonon thermal } \\
\text { conductivity }(\mathrm{W} / \mathrm{mK})\end{array}$ \\
\hline \hline 0 & 6.455 & 8.267 & 2.34 \\
2 & 6.441 & 8.319 & 2.08 \\
4 & 6.433 & 8.352 & 2.04 \\
\hline
\end{tabular}

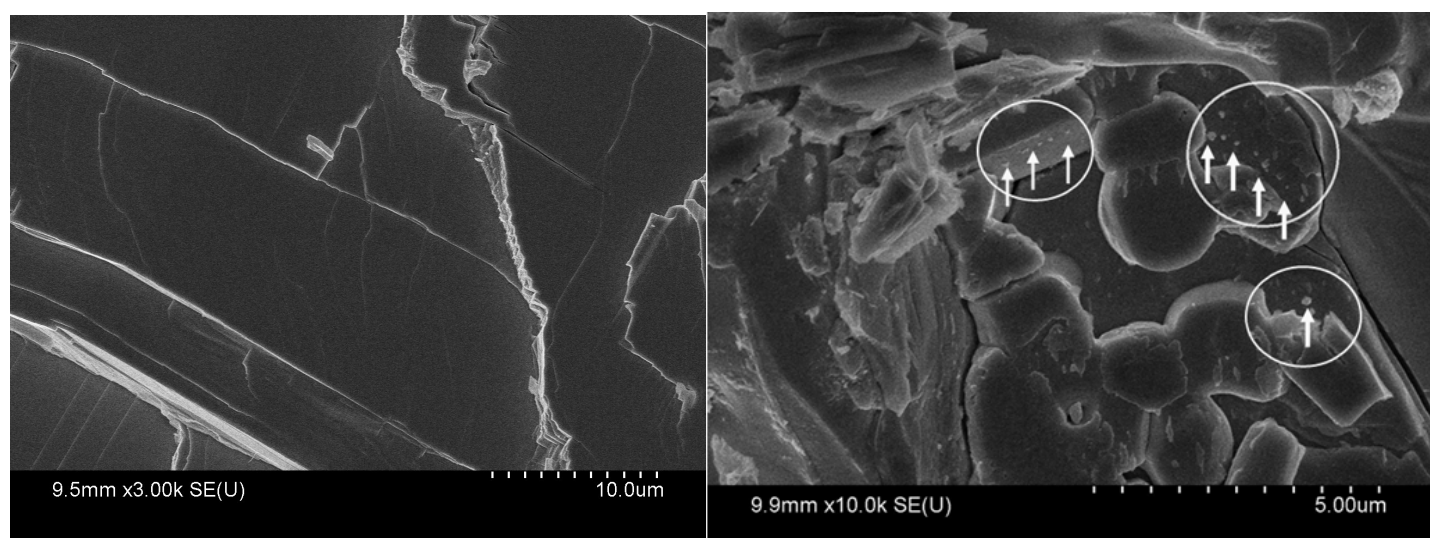

(a)

(b)

Fig. 2. SEM images of a fractured surface of PbTe prepared at (a) ambient pressure, (b) high pressure.

$4 \mathrm{GPa}$ ). As can be seen from the images, the grain size in the sample prepared under high pressure is smaller than that of PbTe prepared at normal pressure. Especially, some second phases are separated and form grains of typically $100 \mathrm{~nm}$ in size, which are distributed over the surface of PbTe prepared at high pressure. According to the energy dispersive $\mathrm{X}$-ray analysis, this nanostructured second phase is $\mathrm{Pb}$. The second $\mathrm{Pb}$ nanophases in $\mathrm{PbTe}$ compounds may effectively enhance phonon scattering and reduce the thermal conductivity.

The temperature dependent electrical resistivity of $\mathrm{PbTe}$ prepared at different pressures is shown in Fig. 3a. The electrical resistivity of the high pressure synthesized samples increases with an increase of temperature. With the increase of pressure, the electrical resistivity of PbTe decreases. It is similar to the result of the in-situ measurement reported by Ovsyannikov [10]. Compared with the data for PbTe samples prepared by other methods $[9,11]$, the electrical resistivity of the samples prepared at high pressure is much lower in the range of measured temperature.
As seen from Fig. 3b, the Seebeck coefficient at room temperature is positive for the sample prepared at normal pressure but the value changes to negative for the sample prepared at high pressure. It indicates that the high pressure can change the type of carriers in $\mathrm{PbTe}$. According to the report of Meng [12], the defects could be produced by high pressure. The formation energy for a certain type of defects is likely to change under stress [13], which leads to a stress-dependent defect concentration. In this study, high pressure is helpful to produce donor defects.

Fig. 4 shows the temperature dependent thermal conductivity for $\mathrm{PbTe}$ samples prepared at $0 \mathrm{GPa}, 2 \mathrm{GPa}$ and $4 \mathrm{GPa}$. The thermal conductivity decreases with an increase in temperature. At the same temperature, the lowest thermal conductivity of the samples is obtained at $2 \mathrm{GPa}$. As it is well known, the thermal conductivity consists of two parts, one is from the phonon thermal conductivity $\kappa_{p h}$ and the other is from the electronic thermal conductivity $\kappa_{\mathrm{e}}$. Here, $\kappa_{\mathrm{e}}$ is expressed by the Wiedemann-Franz law, $\kappa_{\mathrm{e}}=\mathrm{L} \sigma \mathrm{T}$, 


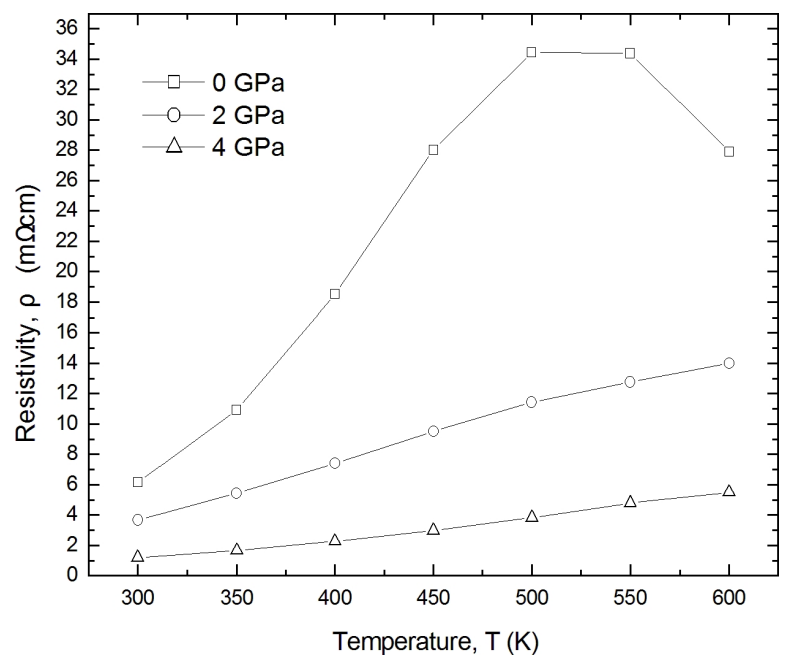

(a)

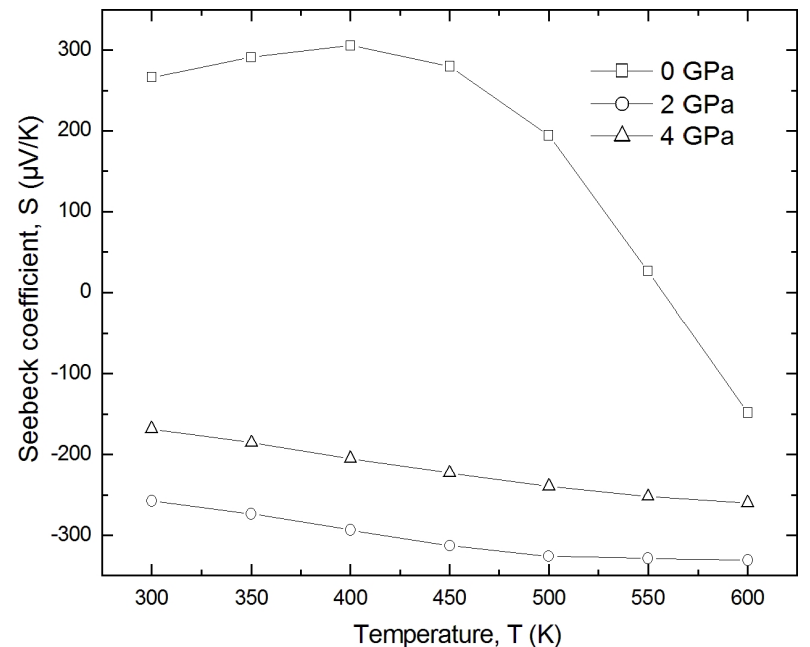

(b)

Fig. 3. Electrical properties of PbTe as a function of temperature, (a) resistivity; (b) Seebeck coefficient.

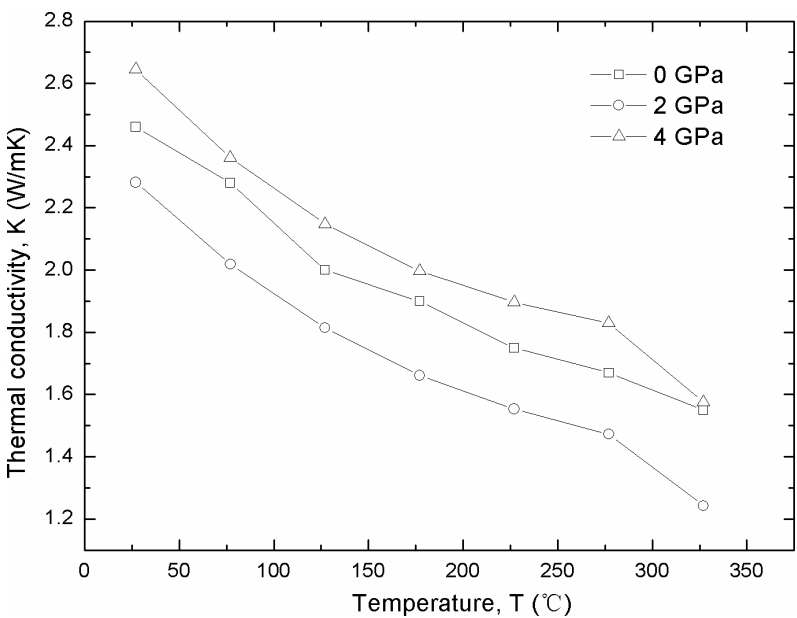

Fig. 4. Thermal conductivity of PbTe as a function of temperature.

where $\mathrm{L}$ is the Lorenz number and $\mathrm{T}$ is the absolute temperature. The value of Lorenz number for PbTe $\left(\mathrm{L}=2.45 \times 10^{-8} \mathrm{~W} \cdot \Omega \cdot \mathrm{K}^{-2}\right)[14]$ is used to estimate $\kappa_{\mathrm{e}}$ at room temperature. As seen form Table 1, the sample prepared at high pressure has lower phonon thermal conductivity than the sample prepared at normal pressure. It could be related to the phonon scattering due to the smaller grain size and the second phase of PbTe created under high pressure.

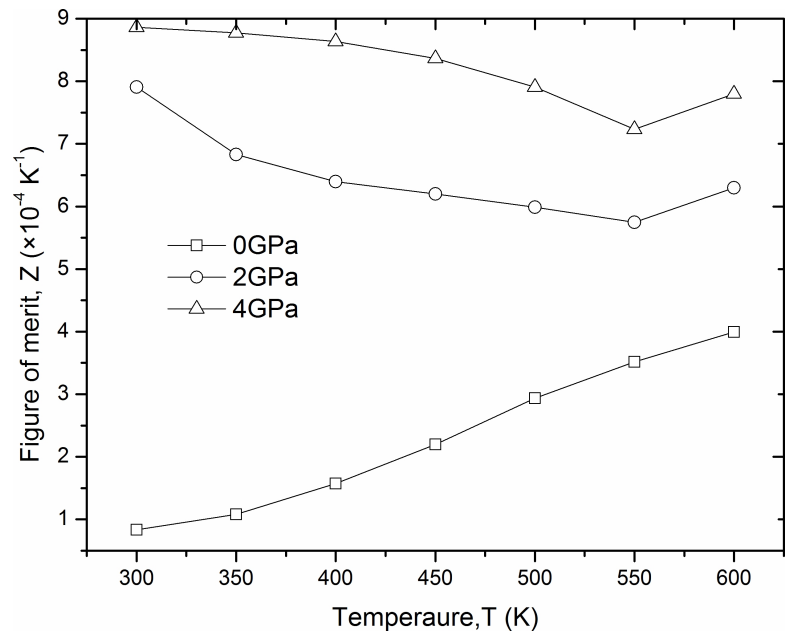

Fig. 5. Figure of merit of $\mathrm{PbTe}$ as a function of temperature.

The temperature dependence of the figure of merit, $Z$, for PbTe calculated from the measured quantities is shown in Fig. 5. It can be seen that the value of $Z$ for PbTe prepared at $0 \mathrm{GPa}$ increases with an increase in temperature. The sample prepared at $4 \mathrm{GPa}$ has the highest value of the figure of merit in the measured temperature range. The maximum value reaches to $8.86 \times 10^{-4} \mathrm{~K}^{-1}$ at room temperature. This value is about twice times higher than that of the sample prepared at ambient pressure $\left(3.99 \times 10^{-4} \mathrm{~K}^{-1}\right)$ and matches to that of 
PbTe alloyed with $\mathrm{Bi}_{2} \mathrm{Te}_{3}$ reported in the literature $\left(7.6 \times 10^{-4} \mathrm{~K}^{-1}\right)$ [15]. A further improved TE performance could be obtained by doping under high pressure.

\section{Conclusions}

Thermoelectric materials $\mathrm{PbTe}$ were prepared by high pressure method successfully. With an increase of temperature, the electrical resistivity and the absolute values of Seebeck coefficient increased. The electrical and thermal properties of $\mathrm{PbTe}$ could be tuned effectively by changing the synthesis pressure. The maximum figure of merit, $\mathrm{Z} \sim 8.86 \times 10^{-4} \mathrm{~K}^{-1}$ was obtained at $300 \mathrm{~K}$ for the sample prepared at $4 \mathrm{GPa}$.

\section{Acknowledgements}

This work was supported by the Opening Project of Henan Key Discipline Open Laboratory of Mining Engineering Materials (MEM1317), The Scientific and Technological Projects under General Administration of Quality Supervision (2012IK049), Open Project of Key Lab of Superhard Materials of Mudanjiang Normal College (201303, 201304), the Project of Science and Technology of Mudanjiang (G2013e1233), Program for Innovative Research Team (in Science and Technology) in the University of Henan Province (2012IRTSTHN007), Foundation of He'nan Educational Committee (14B0006)

\section{References}

[1] Disalvo F., Science, 285 (5428) (1999), 703.
[2] Goldsmid H., Electronic Refrigeration, Pion, London, 1986, p. 10.

[3] RowE D., CRC Handbook of Thermoelectrics, CRC Press, New York, 1995.

[4] Badding J., Annu. Rev. Mater. Res., 28 (1998), 631.

[5] Zhu P., Imaiy., Isoda Y., Shinohara Y., Jia X., Ren G., Zou G., Mater. Trans., 45 (11) 2004, 3102.

[6] Zhu P., Jia X., Chen H., Chen L., Li D., Guo W., Ma H., Ren G., Zou G., Chinese J. High Pressure Phys., 16 (3) (2002), 161.

[7] McGuire M., Maik A., Disalvo F., J. Alloy. Compd., 460 (2008), 8.

[8] Mahan G., Solid State Phys., 51 (1998), 81.

[9] Pei Y., LiU Y., J. Alloy. Compd., 514 (2012), 40.

[10] Ovsyannikov S., Shchennikov V., Appl. Phys. Lett., 90 (2007), 122103.

[11] Li H., Cai K., Wang H., Wang L., Yin J., ZhOU C., J. Solid State Chem., 182 (4) (2009), 869.

[12] Meng J., Polvani D., Jones C., Disalvo F., Fei Y., BADding J., Chem. Mater. 12 (2000), 197.

[13] Godwal B., Jayaraman A., Meenakshi S., Rao R., Sikka S., Vijayakumar V., Phys. Rev. B, 57 (1998), 773.

[14] Shekar N., Polvani D., Meng J., Badding J., Physica B, 358 (2005), 14.

[15] Zhu P., Imai Y., Isoda Y., Shinohara Y., Jia X., Zou G., Chinese Phys. Lett., 22 (2005), 2103. 